\title{
Overexpression of KIAA1199 is an independent prognostic marker in laryngeal squamous cell carcinoma
}

\author{
Meixiang Huang ${ }^{1}$, Feifei Liao ${ }^{2}$, Yexun Song ${ }^{1}$, Gang Zuo $^{3}$, Guolin Tan ${ }^{1}$, Ling Chu ${ }^{\text {Corresp., } 2 \text {, Tiansheng Wang }}{ }^{\text {Corresp. } 1}$ \\ ${ }^{1}$ Department of Otolaryngology Head and Neck Surgery, The Third Xiangya Hospital, Central South University, Changsha, Hunan, China \\ 2 Department of pathology, The Third Xiangya Hospital, Central South University, Changsha, Hunan, Chian \\ 3 Ministry of Education (Central South University), Key Laboratory of Metallogenic Prediction of Nonferrous Metals and Geological Environment Monitoring, \\ Changsha, Hunan, China \\ Corresponding Authors: Ling Chu, Tiansheng Wang \\ Email address: 1799417560@qq.com, tswheaven@hotmail.com
}

Background: KIAA1199 is a recently identified novel gene that is up regulated in various human cancers with poor survival, but its role and the underlying mechanisms in laryngeal squamous cell carcinoma remain unknown. Here, we collected tissues from 105 cases of laryngeal squamous cell carcinoma (LSCC) to investigate the relationships between KIAA1199 protein expression and clinical factors.

Methods: Western blotting and quantitative real-time PCR (qPCR) were utilized to detect the protein and mRNA expression of KIAA1199 in LSCC tissue. Immunohistochemistry (IHC) staining was used to detect the expression of KIAA1199. Patient clinical information, for instance sex, age, pathological differentiation, clinical region, T stage, N stage, clinical stage, operation type, neck lymph dissection, smoking status, and drinking status, were recorded. Kaplan-Meier survival analysis and Cox analysis were applied to find out the relationship between KIAA1199 and LSCC.

Results: Western blotting results showed KIAA1199 protein was significantly higher in tumor tissues vs adjacent non cancerous tissues $(0.9385 \pm 0.1363$ vs1.838 $\pm 0.3209, \mathrm{P}=0.04)$. It revealed that KIAA1199 mRNA expression was considerably higher in tumor tissues $(P<0.001)$ than in adjacent non cancerous tissues by qPCR. IHC results showed, the up-regulated KIAA1199 expression, which was related with some severe clinicopathological parameters: pathologic differentiation $(P, 0.002)$, T stage $(P<0.001), N$ stage $(P<0.001)$, clinical stage $(P<0.001)$, survival time $(P, 0.008)$ and survival status $(P<0.001)$. KaplanMeier survival analysis revealed that patients with high KIAA1199 protein expression had poor overall survival(OS) $(\mathrm{P}<0.05)$. Cox analysis suggested that the KIAA1199 protein expression was an independent prognostic marker for LSCC patients $(P<0.001)$.

Conclusion: Our findings revealed that KIAA1199 protein expression may be used to predict LSCC patient outcome. 


\section{Overexpression of KIAA1199 is an independent}

\section{2 prognostic marker in laryngeal squamous cell}

\section{3 carcinoma}

4

7

8

Meixiang Huang ${ }^{1}$, Feifei $\mathrm{Liao}^{2}$, Yexun Song${ }^{3}$, Gang Zuo ${ }^{4}$, Guolin Tan ${ }^{5}$, Ling $\mathrm{Chu}^{6}$, Tiansheng Wang $^{7}$

1 Department of Otolaryngology Head and Neck Surgery, The Third Xiangya Hospital, Central South University, Changsha, Hunan, P.R. China

2 Department of pathology, The Third Xiangya Hospital, Central South University, Changsha, Hunan, P.R. China

3 Department of Otolaryngology Head and Neck Surgery, The Third Xiangya Hospital, Central South University, Changsha, Hunan, P.R. China

4 Ministry of Education (Central South University), Key Laboratory of Metallogenic Prediction of Nonferrous Metals and Geological Environment Monitoring, Changsha, Hunan, P.R. China 5 Department of Otolaryngology Head and Neck Surgery, The Third Xiangya Hospital, Central South University, Changsha, Hunan, P.R. China

6 Department of pathology, The Third Xiangya Hospital, Central South University, Changsha, Hunan, P.R. China

7 Department of Otolaryngology Head and Neck Surgery, The Third Xiangya Hospital, Central South University, Changsha, Hunan, P.R. China

Corresponding Author:

Tiansheng Wang ${ }^{1}$, Ling $\mathrm{Chu}^{2}$

1Department of Otolaryngology Head and Neck Surgery, The Third Xiangya Hospital, Central South University, Changsha, Hunan, P.R. China

Email address: tswheaven@hotmail.com

2 Department of pathology, The Third Xiangya Hospital, Central South University, Changsha, Hunan, P.R. China

Email address: 1799417560@qq.com 


\section{Abstract}

36

37

38

39

40

41

42

43

44

45

46

47

48

49

50

51

52

53

54

55

56

57

58

59

60

61

62

63

64

65 66

67 5

Background: KIAA1199 is a recently identified novel gene that is upregulated in various human cancers with poor survival, but its role and the underlying mechanisms in laryngeal squamous cell carcinoma remain unknown. Here, we collected tissues from 105 cases of laryngeal squamous cell carcinoma (LSCC) to investigate the relationships between KIAA1199 protein expression and clinical factors.

Methods: Western blotting and real time quantitative PCR (RT- PCR) were used for detect the protein and mRNA expression of KIAA1199 in LSCC tissue. Immunohistochemistry (IHC) staining was used to detect the expression of KIAA1199. Patient clinical information, for instance sex, age, pathological differentiation, clinical region, $\mathrm{T}$ stage, $\mathrm{N}$ stage, clinical stage, operation type, neck lymph dissection, smoking status, and drinking status, were recorded. Kaplan-Meier survival analysis and Cox analysis were applied to identify the relationship between KIAA1199 and LSCC.

Results: Western blotting results showed KIAA1199 protein was significantly higher in tumor tissues vs adjacent non cancerous tissues $(0.9385 \pm 0.1363$ vs $1.838 \pm 0.3209, \mathrm{P}=0.04)$. The KIAA1199 mRNA expression was considerably higher in tumor tissues $(\mathrm{P}<0.001)$ than in adjacent non cancerous tissues by RT-PCR. IHC results showed up-regulated KIAA1199 expression was related with some severe clinicopathological parameters: pathologic differentiation (P, 0.002), $\mathrm{T}$ stage $(\mathrm{P}<0.001)$, $\mathrm{N}$ stage $(\mathrm{P}<0.001)$, clinical stage $(\mathrm{P}<0.001)$, survival time $(\mathrm{P}, 0.008)$ and survival status $(\mathrm{P}<0.001)$. Kaplan-Meier survival analysis showed that patients with high KIAA1199 protein expression had poor overall survival(OS) $(\mathrm{P}<0.05)$. Cox analysis suggested that the KIAA1199 protein expression constituted an independent prognostic marker for LSCC patients $(\mathrm{P}<0.001)$.

Conclusion: Our findings revealed that KIAA1199 protein expression may be used to predict LSCC patient outcome.

Keywords: KIAA1199, CEMIP, Laryngeal squamous carcinoma, Prognostic marker

\section{Introduction}


68

69

70

71

72

73

74

75

76

77

78

79

80

81

82

83

84

85

86

87

88

89

90

91

92

93

94

95

96

97

98

99

100

101

Laryngeal squamous cell carcinoma is the most common laryngeal cancer, and it is the second highest incidence among head and neck cancer(Ling Gao, et al., 2018). In 2016, Laryngeal malignancies accounted for approximately 13,400 cancer cases in U.S.A, of which an estimated 3600 patients succumbed to the disease(Rebecca L. Siegel, et al., 2019). The number of laryngeal cancer has radically changed in the last 20 years in USA(S. Michael Rothenberg, et al., 2012). In the available treatments, there have been improvements, but the patients still need identified novel gene that is up-regulated in human cancer suffer from poor prognosis. So, in the pathogenesis of LSCC, identification of the key molecules is urgently needed to improve the treatment of LSCC.

The KIAA1199 gene, which was first discovered in association with non-syndromic hearing loss(Satoko Abe, et al., 2003). Nowadays, we have known that the KIAA1199 gene is expressed in a wide range of normal human tissues(Yongsheng Zhang, et al., 2014). Over-expression of KIAA1199 contributes to resistance to cell immortalization and cancerization in normal human cells and is associated with cell death(Eriko Michishita, et al., 2006). Several researches have illuminated that KIAA1199 is over-expressed in different cancers, including oral squamous cell carcinoma(Pitak Chanthammachat, et al., 2013), breast cancer(Nikki A. Evensen, et al., 2013), gastric cancer(Shinji Matsuzaki, et al., 2009), colorectal tumors((K Birkenkamp-Demtroder, et al., 2011; Lawrence C. LaPointe, et al., 2012), prostate cancer(Eriko Michishita, et al., 2006), ovarian cancer(Fan Shena, et al., 2019) and hepatocellular carcinoma(Zhengchen Jiang, et al., 2018). The workings proved, that KIAA1199, which regulates the proliferation, migration, and invasion of tumors. So far, in LSCC remain an enigma, we have no report about KIAA1199 expression in LSCC, and the clinical value and biological role of KIAA1199.

We implemented immunohistochemical detection of KIAA1199 protein expression to investigate the clinical significance of KIAA1199 and to detect whether it plays a key role in the progression of LSCC in 105 paired formalin-fixed and paraffin-embedded cancer and adjacent noncancer tissues obtained from patients with LSCC. In the end, we illuminated the clinicopathologic characteristics of LSCC patients was related to KIAA1199, which were statistically evaluated.

\section{Materials \& Methods}

\section{Patient enrolment and arrange follow up}

The research was implemented in the Department of ENT, the Third Xiangya Hospital, Central South University. We collected 10 pairs of fresh specimens and their matched adjacent non cancerous specimens, which were from patients diagnosed with human laryngeal squamous cell carcinoma by pathological examination in February 2018. A total of 105 patients who had been

Peer] reviewing PDF | (2020:01:44662:2:0:NEW 29 Jun 2020) 
102 performed curative resection for LSCC were registered from 2009 to 2014. Patients with 103 recurrence of laryngeal cancer or multiple cancers were excluded. No anticancer therapy was given

104

105

106

107

108

109

110

111

112

113

114

115

116

117

118

119

120

121

122

123

124

125

126

127

128

129

130

131

132

133

134

135

136

before surgery. Postoperative pathological examination of patients diagnosed with laryngeal squamous cell cancer. Patient clinical data such as sex, age, pathological differentiation, clinical region, T stage, $\mathrm{N}$ stage, clinical stage, operation type, neck lymph dissection, smoking status, and drinking status were collected. To investigate the prognostic value of KIAA1199 in postoperative patients, we examined the overall survival rate (OS) of the LSCC patients. The average arrange follow up cycle was 54 months ( 5 months extent to 10 years). Prior to the start of the study, we obtained the written informed consent of all patients and the approval of the hospital's Human Research Ethics Committee in accordance with the Helsinki Declaration Guidelines(NO. 2018S084). All tissue samples were treated and anonymous in accordance with ethical and legal standards. The tumor stage was determined according to the TNM(tumor, lymph node, metastasis) grading of the International Union Against Cancer (UICC, 2002).

\section{RNA extraction and real-time RT-PCR}

According to the manufacturer's protocol, the total RNA was isolated from LSCC and matched adjacent tissues by using TRIzol Reagent (Invitrogen). Nanodroplet spectrophotometer (Thermo Scientific, Waltham, MA, USA) was used to measure the concentration and purity of total RNA. According to the manufacturer's instructions, the total RNA was converted to cDNA using a quantitative PCR (qPCR) reverse transcription kit (TOYOBO Life Science, Shanghai, P.R. China), fresh tissues were used to synthesize cDNA. Real-time RT-PCR was applied three times using a KOD SYBR qPCR Mix Fluorescent Quantitative PCR kit (TOYOBO Life Science, Shanghai, P.R. China). PCR and data collection were conducted by using an EP Real-time PCR System (Eppendorf Inc., Hauppauge, NY, USA). For standardization, we used GAPDH as an endogenous control. The primers used in our study were purchased from Sangon Biotech (Shanghai, P.R. China), and the following primer sequences were used: KIAA1199, F primer 5'CCAGTAACCTGCGAATGAAGA-3' and R primer 5'-TGGTCCCAGTGGATGGTGTAG-3'. GAPDH, F primer 5'-TTGGTATCGTGGAAGGACTCA-3' and R primer 5'TGTCATCATATTTGGCAGGTT-3'. The reaction conditions were $95^{\circ} \mathrm{C}$ for $5 \mathrm{~min}$, followed by 40 cycles at $95^{\circ} \mathrm{C}$ for $15 \mathrm{sec}$ and $58^{\circ} \mathrm{C}$ for $30 \mathrm{sec}$. The relative expression level was determined by the $2-\Delta \Delta \mathrm{Ct}$ method.

\section{Western Blotting Analysis}

Proteins were extracted from LSCC fresh tissue samples and adjacent non cancerous fresh tissue samples. The Western blotting analysis was carried out according to our previous 
137

138

139

140

141

142

143

144

145

146

147

148

149

150

151

152

153

154

155

156

157

158

159

160

161

162

163

164

165

166

167

168

169

170

171

172

173

article(Wei Li, et al., 2012).Primary antibodies were used as follows: polyclonal rabbit antiKIAA1199 antibody (diluted 1:1,000), anti-GAPDH antibody (diluted 1:5000), and horseradish peroxidase-conjugated secondary antibody $(1: 10,000)$.

\section{Immunohistochemistry}

One hundred and five formalin-fixed, paraffin-embedded LSCC tissues were used for the immunohistochemistry (IHC) studies. Briefly, the tissue was sliced continuously into approximately $4 \mu \mathrm{m}$ section, paraffin was removed from the sections using a graded alcohol series of $100 \%$ and $95 \%$ in xylene, rehydrated in $75 \%$, and finally washed with PBS. Subsequently, the antigen was prepared with sodium citrate buffer PBS and incubated in 3\% $\mathrm{H} 2 \mathrm{O} 2$ deionized water for 15 minutes to inactivate endogenous peroxidase. The sections were washed 3 times with PBS, incubated with calf serum to block non-specific antigen for $10 \mathrm{~min}$, incubated with polyclonal rabbit anti-KIAA1199 antibody (1:70) at room temperature for 1 hour, washed with PBS three times, and then incubated with secondary antibody at room temperature for 30 minutes. Sections were washed with PBS 3 times, stained with diaminobenzidine (DAB) for 4 minutes, washed 3 more times with PBS, restained with haematoxylin for 30 seconds, washed with flowing water, dried and sealed. Dried sections were observed with an optical microscope. The positive control was gastric cancer tissue confirmed by pathological examination, and the adjacent normal tissues from patients with LSCC were used as the negative control.

The positive expression of KIAA1199 was patchy with aggregates of brown granules in the cytoplasm. Semiquantitative analysis was used to determine the percentage of positive cells under the microscope and score the staining intensity. Two senior pathologists of the Department of Pathology were assigned to read the slides in a double-blinded manner, and 3-5 different fields were randomly selected from each IHC staining section for observation. The staining results were semiquantitatively analysed in terms of staining intensity and percentage of cells with positive expression. Evaluation of dyeing intensity: range, 0-3; colourless (negative) $=0$, weak (pale yellow $)=1$, medium (brown-yellow) $=2$, strong $(\tan )=3$. Percentage of stained cells: range, $0-3$; percent positive cells $<5 \%=0,0.5 \%-10 \%=1,10 \%-50 \%=2, \geq 50 \%=3$. The score of the two was multiplied to show the positive grade: 0 is negative $(-), \leq 3$ is low expression, and $>3$ is higher expression.

\section{Statistical analysis}

Our results were interpreted with GraphPad Prism version 7.0 (GraphPad Software, Inc., La Jolla, CA, USA) and SPSS 23.0 software package (SPSS, 112 Y.-H. HAO ET AL. Chicago, IL, USA). Chi-square test was used to analyze the associate with KIAA1199 protein expression and clinicopathological characteristics in LSCC patients. Cox regression analysis estimated the risk of 
174 death associated with KIAA1199 protein expression. Kaplan-meier method was used to analyze 175 the total survival curve. Other data were analysed using Student's t-test, and ANOVA was 176 conducted to determine the differences in two or more groups. All data are presented as the mean $177 \pm \mathrm{SD}$ with $\mathrm{P}<0.05(* \mathrm{P}<0.05, * * \mathrm{P}<0.01, * * * \mathrm{P}<0.005, * * * * \mathrm{P}<0.001)$.

178

179

180

181

182

183

184

185

186

187

188

189

190

191

192

193

194

195

196

197

198

199

200

201

202

203

204

205

206

207

208

209

210

\section{Results}

\section{Clinical data}

To understand the clinical features of patients, the detailed data of the patients, such as sex, age, pathological differentiation, clinical region, $\mathrm{T}$ stage, $\mathrm{N}$ stage, clinical stages, operation type, neck lymph dissection, smoking status, and drinking status, were collected from their medical records, and these data are summarized in Table 1 for the 105 patients in this study; 103 (98.1\%) were men, and $2(1.9 \%)$ were women, ranging in age from 37 to 82 years. T1-T2 stage was detected in 70 patients $(66.6 \%), \mathrm{N} 1-\mathrm{N} 3$ stage was detected in 20 patients $(19 \%)$, and the overall survival (OS) time ranged from 6 to 108 months.

\section{Increased Expression of KIAA1199 in Human LSCC tissues}

To uncover the role of KIAA1199 expression in LSCC, we first detected KIAA1199 protein and mRNA expression in 10 pairs of fresh human LSCC specimens and their matched adjacent noncancerous specimens using Western blotting (Fig. 1A, 1B), IHC (Fig. 2) and RT-PCR (Fig. 1C). As shown in, KIAA1199 protein levels were significantly higher in LSCC tissues (1.838 \pm 0.3209 vs $0.9385 \pm 0.1363, \mathrm{P}=0.04$ ) (Fig.1B) than in adjacent noncancerous tissues. RT-PCR revealed that KIAA1199 mRNA expression was considerably lower in adjacent noncancerous tissues $(\mathrm{P}<0.001)$ than in cancer tissues (Fig. 1C). Then, we compared the expression of KIAA1199 in 105 LSCC tissues and their adjacent noncancerous tissues through immunohistochemistry. There was weak or negative expression of KIAA1199 in adjacent noncancerous tissue but high expression in the cytoplasm of LSCC tissue cells. The positive staining and negative staining rates in LSCC tissues were 52.4\% (55/105, Table 1) and 47.6\% (50/105), respectively. Semiquantitative analysis showed that KIAA1199 was significantly increased in LSCC tissues. Representative photographs of the immunostaining are shown in figure 3 A-F. RT-PCR results for KIAA1199 mRNA levels were agree with the Western blotting and IHC results, showing that KIAA1199 is increased in LSCC tissues.

\section{KIAA1199 expression is associated with pathologic differentiation, $\mathrm{T}, \mathrm{N}$, clinical stage, survival status and survival times of LSCC}

Peer] reviewing PDF | (2020:01:44662:2:0:NEW 29 Jun 2020) 
211 In order to further reveal the character of KIAA1199 in LSCC, we evaluated the relationship 212 between its expression and the clinicopathological characteristics of LSCC. As shown in Table 2, 213 upregulation of KIAA1199 expression was associated with some clinicopathological parameters: 214 pathologic differentiation $(\mathrm{P}, 0.002)$, $\mathrm{T}$ stage $(\mathrm{P}<0.001)$, $\mathrm{N}$ stage $(\mathrm{P}<0.001)$, clinical stage $215(\mathrm{P}<0.001)$, survival time $(\mathrm{P}, 0.008)$ and survival status $(\mathrm{P}<0.001)$. However, KIAA1199 expression 216 was not correlated with age, sex, clinical region, smoking status, or drinking status.

217

218

219

220

221

222

223

224

225

226

227

228

229

230

231

232

233

234

235

236

237

238

239

240

241

242

243

244

245

246

247

248

\section{Survival assessment: A high level of KIAA1199 is predictive of poor prognosis in LSCC patients}

The survival curve was plotted by kaplan-meier method, and the survival time was tested by log-rank test. The results showed that LSCC patients with high KIAA1199 expression had a lower prognosis, and low KIAA1 199 expression in LSCC patients $(\mathrm{P}<0.001$, for OS) was related with considerably longer OS compared with high KIAA1199 expression. The median OS for high KIAA1199 expression was $60 \pm 4.113$ months and that for low KIAA1199 expression was $96 \pm 7.928$ months (Fig. $3 \mathrm{G}$ ). Then analyzed independent prognostic factors for survival in patients with LSCC by using univariate and multivariate Cox proportional hazards analysis. The univariate analysis results (Table 3 ) showed that age $(\mathrm{HR}=1.032,95 \% \mathrm{CI}: 1.001-1.063 ; \mathrm{P}, 0.04)$, pathologic differentiation $(\mathrm{HR}=0.643,95 \% \mathrm{CI}: 0.524-0.789 ; \mathrm{P}<0.001)$, T stage $(\mathrm{HR}=1.402,95 \% \mathrm{CI}: 1.139$ - 1.724; $\mathrm{P}<0.001)$, N stage $(\mathrm{HR}=1.679,95 \% \mathrm{CI}: 1.148$ - 2.4577; P, 0.008), clinical stage (HR $=1.445,95 \%$ CI: 1.180 - 1.769; $\mathrm{P}<0.001)$, operation type $(\mathrm{HR}=0.380,95 \% \mathrm{CI}: 0.222$ - 0.650; $\mathrm{P}<0.001)$ and KIAA1199 expression $(\mathrm{HR}=12.165$, 95\% CI: $5.434-27.233 ; \mathrm{P}<0.001)$ were significantly associated with the overall survival of LSCC patients. Multivariate survival analysis (table 4) showed that KIAA1199 expression was statistically significant predictor of OS(HR $=27.937,95 \% \mathrm{CI}: 10.600-73.632 ; \mathrm{P}<0.0001)$ and that age $(\mathrm{HR}=1.039,95 \% \mathrm{CI}: 1.003$ - 1.077; $\mathrm{P}$, $0.0354)$, clinical stage $(\mathrm{HR}=0.704,95 \% \mathrm{CI}: 0.581-0.960 ; \mathrm{P}, 0.023)$, operation type $(\mathrm{HR}=0.285$, 95\% CI: 0.093-0.870; P, 0.027), T stage (HR =0.68, 95\% CI: 0.529-0.874; P, 0.003) and smoking status $(\mathrm{HR}=0.19,95 \% \mathrm{CI}: 0.057-0.630 ; \mathrm{P}, 0.007)$ were independent predictive factors for OS.

\section{Discussion}

In order to identify novel gene that is up-regulated in human cancer with poor prognosis, a deeply cognition to the molecular biology profiles of LCSS is a vital work. To outcomes remain elusive, the molecular pathways involved in LSCC incidence, progression and clinical yet. Espeially the endoplasmic reticulum, KIAA1199, which is a glycosylated protein which located in the cytoplasm and membrane(Amit Tiwari, et al., 2013; Eriko Michishita, et al., 2006; Nikki A. Evensen, et al., 2013). The relationship between cancer and KIAA1199 have been studied in many research directions. KIAA1199 is a recently identified novel gene that can regulate cell growth 
249

250

251

252

253

254

255

256

257

258

259

260

261

262

263

264

265

266

267

268

269

270

271

272

273

274

275

276

277

278

279

280

281

282

283

284

and invasion and could be a new therapeutic target in breast cancer(Mohammad-Saeid Jami, et al., 2014). A similar analysis reported, KIAA1 199 overexpression can predict poor survival in patients with colon cancer(Jian Xu, et al., 2015). By several mechanisms, KIAA1199 protein can accelerate cancer progression. Simultaneously, other research have shown that the KIAA1199 protein expression level is elevated upon p53 activation(Shinji Matsuzaki, et al., 2009). KIAA1199 is also related to angiogenesis in rheumatoid arthritis(Xinyu Yang, et al., 2015). However, the mechanism of KIAA1 199 tumor-promoting effects in LSCC is little known.

In our study, we first verified KIAA1199 protein and mRNA expression in 10 pairs of fresh surgically resected LSCC samples by Western blotting, IHC and real-time RT-PCR. Our results have drawn a conclusion that KIAA1199 was highly expressed in LSCC cancerous in contrast to adjacent non cancerous tissue. In view of our data, we also can censor the hidden expression of KIAA1199 by immunohistochemistry in 105 paraffin-embedded sections (2009-2014) to further explore the relationship between KIAA1199 and clinicopathological characteristics. By our data analysis, showed that KIAA1199 expression was not kenspeckle related with clinical parameters which as age, sex, clinical region, smoking, or drinking. Interestingly, for some severe clinicopathological parameters: pathologic differentiation $(\mathrm{P}, 0.002), \mathrm{T}$ stage $(\mathrm{P}<0.001), \mathrm{N}$ stage $(\mathrm{P}<0.001)$, clinical stage $(\mathrm{P}<0.001)$, survival time $(\mathrm{P}, 0.008)$ and survival status $(\mathrm{P}<0.001)$, the significant correlations were observed. Through our experiments, we obtained many data, which provides a new evidence that KIAA1199 is highly expressed in primary LSCC tissues and its immunoreactivity is higher in cancerous than adjacent noncancerous tissues, revealing that KIAA1199 might help distinguish benign from malignant larynx tumors. Moreover, our results and analysis illuminated that the expression of KIAA1199 was elevated in LSCC tissues with aggressive clinicopathological characteristics, suggesting its potential as a marker of cancer invasionality.

The abnormal expression of KIAA1199 has also been found in other cancer studies, such as oral squamous cell carcinoma(Pitak Chanthammachat, et al., 2013), breast cancer(Nikki A. Evensen, et al., 2013), gastric cancer(Shinji Matsuzaki, et al., 2009), colorectal tumors(Amit Tiwari, et al., 2013; K Birkenkamp-Demtroder, et al., 2011; Lawrence C. LaPointe, et al., 2012), prostate cancer(Eriko Michishita, et al., 2006), ovarian cancer(Fan Shena, et al., 2019) and hepatocellular carcinoma(Zhengchen Jiang, et al., 2018). It was reported(Xuehua Jiao, et al., 2019) that KIAA1199 was abnormaly increased in the papillary thyroid tumor compared with normal specimens tissues and that upregulation of KIAA1199 was positively correlated with more advanced clinical variables. There was analysis showed that the cell invasion and migration were related with KIAA1199. KIAA1199 silencing inhibited the invasive ability of papillary thyroid cancer cells by affecting epithelial-mesenchymal transition (EMT) in vitro and in vivo. Additionally, the same as our study, In clone cancer study(Jian Xu, et al., 2015) proved the

Peer) reviewing PDF | (2020:01:44662:2:0:NEW 29 Jun 2020) 
285 expression of KIAA1199 was also observably associated with tumor invasion, metastasis and 286 TNM staging. Increased mortality risks associated with overexpression of KIAA1199 in primary 287 hepatocellular cancer patient. Previous researches have demonstrated that up-regulation of

288

289

290

291

292

293

294

295

296

297

298

299

300

301

302

303

304

305

306

307

308

309

310

311

312

313

314

315

316

317 KIAA1199 motivates carcinogenesis, motility and apoptosis. Metastasis, invasion, and cell movement of a variety of cell types are associated with KIAA1199 expression(Yongsheng Zhang, et al., 2014). By the Wnt/ $\beta$-catenin signalling pathway, EMT is one of the important processes mediated, which plays a key role in cancer invasion and metastasis(Yanyuan Wu, et al., 2012). Interestingly, the KIAA1199 signalling pathway also induces the development and progression of tumor. Other researches showed that the cell proliferation and mobility of colorectal cancer cells were inhibited by knocking down the expression of CEMIP in vitro, and the EMT process of colorectal cancer cells is suppressed by shRNA-CEMIP via inactivation of the Wnt/ $\beta$-catenin/Snail pathway(Guodong Liang, et al., 2018). Collectively, our results demonstrated that the overexpression of KIAA1199 mRNA may affect tumor spread, lymph node metastasis, tumor differentiation and prognosis(Shinji Matsuzaki, et al., 2009). In a report, it was defined KIAA1199 as an carcinogenic protein induced by HPV infection and compositive NF-kB activity that transmits pro-survival and aggressive signals via EGFR signalling(Kateryna Shostak, et al., 2014). Research has suggested that KIAA1199 may promote the development of ovarian cancer by regulating PI3K/AKT signalling(Fan Shena, et al., 2019). One study insisted, AMPK/GSK3 $\beta / \beta$ catenin cascade triggered KIAA1199 over-expression may promote migration and invasion in anoikis-resistant prostate cancer cells by increasing PDK4-associated metabolic reprogramming, which may provide a novel therapeutic target for the prostate cancer(Peng Zhang, et al., 2018). Therefore, in the light of the upper research about the KIAA1199-related signalling pathway, we can draw a conclusion that KIAA1199 can influence the occurrence and development of laryngeal cancer, which may also be related to the Wnt/ $\beta$-catenin, EGFR, PI3K/AKT, and AMPK/GSK3 signalling pathways and other pathways. Thence, we will carry out a molecular mechanism research of KIAA1199 in LSCC cells and animal models in our future study.

Some limitations exist in our research. First, the sample size of this study was a little small. As a retrospective study design that the selection bias might not be ignored. Second, our study did not explore the effect of other treatments for LSCC on the prognosis of patients, such as radiotherapy and chemotherapy. So, in the future studies, we will carry out cell biology experiments to verify our findings, such as gene transfection and cell migration assays. 
319

320

321

322

323

324

325

326

\section{7}

328

329

330

331

332

333

334

335

336

337

338

339

340

341

342

343

344

345

346

347

348

In conclusion, our results revealed significant associations of KIAA1199 protein expression with various clinicopathologic characteristics and the prognosis of LSCC patients. Moreover, survival analysis illuminated KIAA1199 was an independent prognostic factor for overall survival in LSCC. All of these findings indicate that the KIAA1199 protein might be used as a pathological marker to identify individuals with poor outcomes and to provide a reference for clinical therapy in the future. Further studies are required to investigate its rationality as a marker and the potential pathways involved in KIAA1199-mediated cell invasion and metastasis.

\section{Acknowledgements}

This study was supported by the Hunan Provincial Science Foundation of China (No 2016JJ4104) and the National natural science foundation of China (NO.81702706).

We thank Dr. Sheng Xiao for assistance with the IHC experiments.

\section{References}

Eriko Michishita GGs, J. Carl Barrett, Izumi Horikawa. 2006. Upregulation of the KIAA1199 gene is associated with cellular mortality. Cancer Lett 239:71-77. DOI 10.1016/j.canlet.2005.07.028.

Fan Shena Z-hZ, Yao Liuc, Shuo Chena, Xiu-jie Shenga, Yang Zhao. 2019. CEMIP promotes ovarian cancer development and progression via the PI3K/AKT signaling pathway. Biomed Pharmacother 114:108787. DOI 10.1016/j.biopha.2019.108787.

Gu Changjiang NQ, Ni Kan, Zhang Shu, Qian Haixin. 2018. Expression and clillical significance of KIAA1199 in primary hepatocellular carcinoma. Natl Med J China 98:1609-1613. DOI 10. 3760/cma. j. issn. 0376-2491. 2018. 20. 017.

Guodong Liang XF, Yubo Yang, Yan Song. 2018. Silencing of CEMIP suppresses Wnt/ $\beta$ catenin/Snail signaling transduction and inhibits EMT program of colorectal cancer cells. Acta Histochemica 120:56-63. DOI 10.1016/j.acthis.2017.11.002.

Jian Xu YL, Xudong Wang, Jianfei Huang, Huijun Zhu, Zhiqian Hu, Defeng Wang. 2015. Association between KIAA1199 overexpression and tumor invasion, TNM stage, and poor prognosis in colorectal cancer. Int J Clin Exp Pathol 8:2909-2918. 
349

350

351

352

353

354

355

356

357

358

359

360

361

362

363

364

365

366

367

368

369

370

371

372

373

374

375

376

377

378

379

K Birkenkamp-Demtroder AM, F Mansilla, K Thorsen, CL Andersen, B Øster, S Hahn, TF Ørntoft. 2011. Repression of KIAA1199 attenuates Wnt-signalling and decreases the proliferation of colon cancer cells. Br J Cancer 105:552-561. DOI 10.1038/bjc.2011.268.

Kateryna Shostak XZ, Pascale Hubert, Serkan Ismail Go“ktuna, Zheshen Jiang, Iva Klevernic, Julien Hildebrand, Patrick Roncarati, Benoit Hennuy, Aure'lie Ladang, Joan Somja, Andre' Gothot, Pierre Close, Philippe Delvenne, Alain Chariot. 2014. NF-kappaB-induced KIAA1199 promotes survival through EGFR signalling. Nat Commun 5:5232. DOI 10.1038/ncomms6232.

Lawrence C. LaPointe SKP, Robert Dunne, Glenn S. Brown, Letitia Pimlott,Snigdha Gaur, Aidan McEvoy, Melissa Thomas, David Wattchow, Peter L. Molloy, Graeme P. Young. 2012. Discovery and validation of molecular biomarkers for colorectal adenomas and cancer with application to blood testing. PLoS One 7:e29059. 10.1371/journal.pone.0029059.

Mohammad-Saeid Jami JH, Miao Liu, Michelle L Varney, Hesham Hassan, Jixin Dong, Liying Geng, Jing Wang, Fang Yu, Xin Huang, Hong Peng, Kai Fu1, Yan Li, Rakesh K Singhand Shi-Jian Ding. 2014. Functional proteomic analysis reveals the involvement of KIAA1199 in breast cancer growth, motility and invasiveness. BMC Cancer 14:194. DOI 10.1186/1471-2407-14-194.

Nikki A. Evensen CK, Hoang-Lan Nguyen, Kevin Zarrabi, Antoine Dufour, Pournima Kadam, You-jun Hu, Ashleigh Pulkoski-Gross, Wadie F. Bahou, Stanley Zucker, Jian Cao. 2013. Unraveling the role of KIAA1199, a novel endoplasmic reticulum protein, in cancer cell migration. J Natl Cancer Inst 105:1402-1416. DOI 10.1093/jnci/djt224.

Peng Zhang YS, Yadong Sun, Xuechao Li, Lifeng Chen, Likun Yang, Yifei Xing. 2018. AMPK/GSK3beta/beta-catenin cascade-triggered overexpression of CEMIP promotes migration and invasion in anoikis-resistant prostate cancer cells by enhancing metabolic reprogramming. FASEB J:fj201701078R. DOI 10.1096/fj.201701078R.

Pitak Chanthammachat WP, Kowit Pruegsanusak, Sittiruk Roytrakul, Chantragan Srisomsap, Daranee Chokchaichamnankit, Jisnuson Svasti, Pleumjit Boonyaphiphat, Singkhamanan K, Paramee Thongsuksai. 2013. Comparative proteomic analysis of oral squamous cell carcinoma and adjacent non-tumour tissue from Thailand. Arch Oral Biol 58:1677-1685. DOI 10.1016/j.archoralbio.2013.08.002. 
380

381

382 383

384

385

386

387

388

389

390

391

392

393

394

395

396

397

398

399

400

401

402

403

404

405

406

407

408

409

410

Rebecca L. Siegel KDM, Ahmedin Jemal, DVM. 2019. Cancer statistics, 2019. CA Cancer J Clin 69:7-34. DOI 10.3322/caac.21551.

S. Michael Rothenberg LWE. 2012. The molecular pathogenesis of head and neck squamous cell carcinoma. J Clin Invest 122:1951-1957. DOI 10.1172/jci59889.

Satoko Abe S-iU, Yusuke Nakamura. 2003. Mutations in the gene encoding KIAA1199 protein, an inner-ear protein expressed in Deiters' cells and the fibrocytes, as the cause of nonsyndromic hearing loss. J Hum Genet 48:564-570. DOI 10.1007/s10038-003-0079-2.

Shinji Matsuzaki FT, Koshi Mimori, Kouichirou Tahara, Hiroshi Inoue, Masaki Mori. 2009. Clinicopathologic significance of KIAA1199 overexpression in human gastric cancer. Ann Surg Oncol 16:2042-2051. DOI 10.1245/s10434-009-0469-6.

Xinyu Yang PQ, Bingbing Chen, Yaoyao Lin, Zhonghao Zhou, Renshan Ge, Hai Zou, Jianmin Wang, Jianguang Wang. 2015. KIAA1199 as a potential diagnostic biomarker of rheumatoid arthritis related to angiogenesis. Arthritis Res Ther 17:140. DOI 10.1186/s13075-015-0637-y.

Xuehua Jiao JY, Xiujie Wang, Xueyan Yin, Guodong Zhang, Xingbo Cheng. 2019. KIAA1199, a Target of MicoRNA-486-5p, Promotes Papillary Thyroid Cancer Invasion by Influencing Epithelial-Mesenchymal Transition (EMT). Med Sci Monit 25:6788-6796. DOI 10.12659/MSM.918682.

Yanyuan Wu CG, Juri Kim, Nicole Mosher, Seyung Chung, Dennis Slamon, Jaydutt V. Vadgama. 2012. Expression of Wnt3 activates Wnt/beta-catenin pathway and promotes EMT-like phenotype in trastuzumab-resistant HER2-overexpressing breast cancer cells. Mol Cancer Res 10:1597-1606. DOI 10.1158/1541-7786.MCR-12-0155-T.

Yongsheng Zhang SJ, WEN G. JIANG. 2014. KIAA1199 and its biological role in human cancer and cancer cells (review). Oncol Rep 31:1503-1508. DOI 10.3892/or.2014.3038.

Zhengchen Jiang XZ, Binyao shi, Dan luo, Bin Jin. 2018. KIAA1199 overexpression is associated with abnormal expression of EMT markers and is a novel independent prognostic biomarker for hepatocellular carcinoma. Onco Targets Ther 11:8341-8348. DOI 10.2147/OTT.S187389.

Wei Li, Guolin Tan, Yanhong Ma, Heqing Li and Guangxiang He. 2012. Inhibition of alpha folate receptor resulting in a reversal of taxol resistance in nasopharyngeal carcinoma. Otolaryngol Head Neck Surg 146:250-258. 10.1177/0194599811426260. 
411

412

413

Peer) reviewing PDF | (2020:01:44662:2:0:NEW 29 Jun 2020) 
Figure 1

Figure 1 The protein of KIAA1199 was overexpression in LSCC tissue specimens.

(A) (B) The protien expression of KIAA1199 in adjacent non cancerous tissue and LSCC tissue by Western bloting.****P<0.001. (C) The mRNA expression of KIAA1199 in adjacent non cancerous tissue and LSCC tissue by RT-PCR. ****P<0.001.
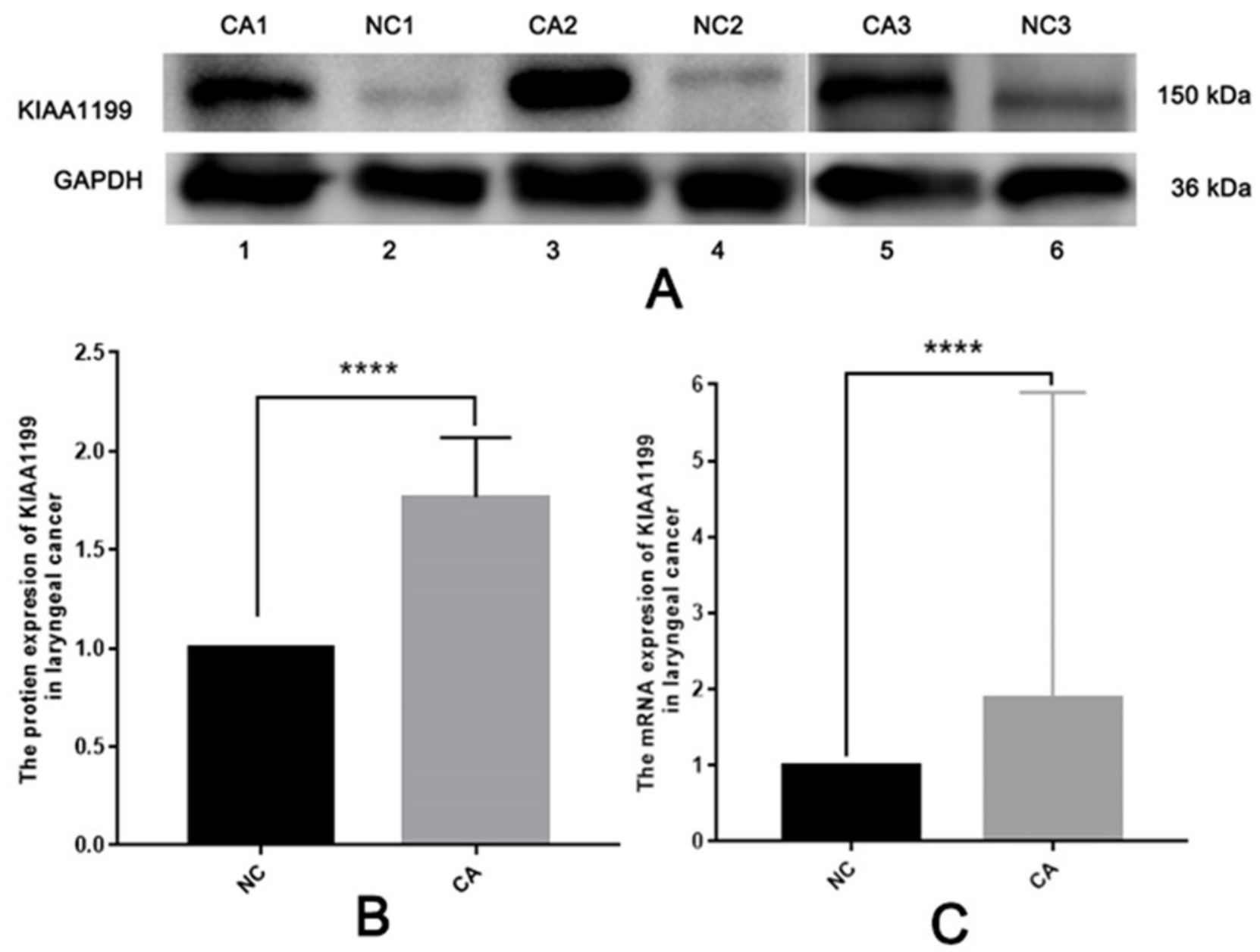


\section{Figure 2}

Figure 2 Representative images of immunohistochemical staining for KIAA1199 expression in larynx specimens.

(A) Negative expression of KIAA1199 in adjacent non cancerous specimens. (B) Low expression of KIAA1199 in LSCC pecimens. (C) High expression of KIAA1199 in LSCC specimens. Original magnification: 40X; scale bars: 20um.

\section{A}

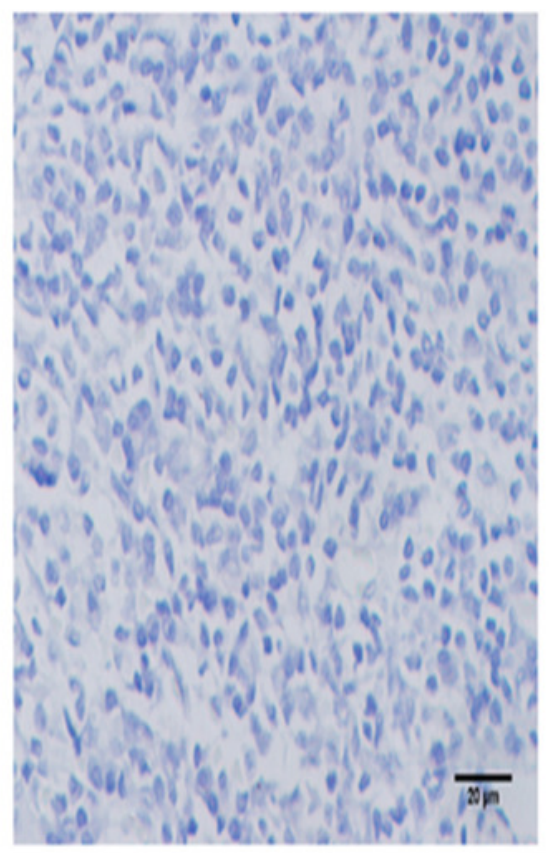

Negative control
B

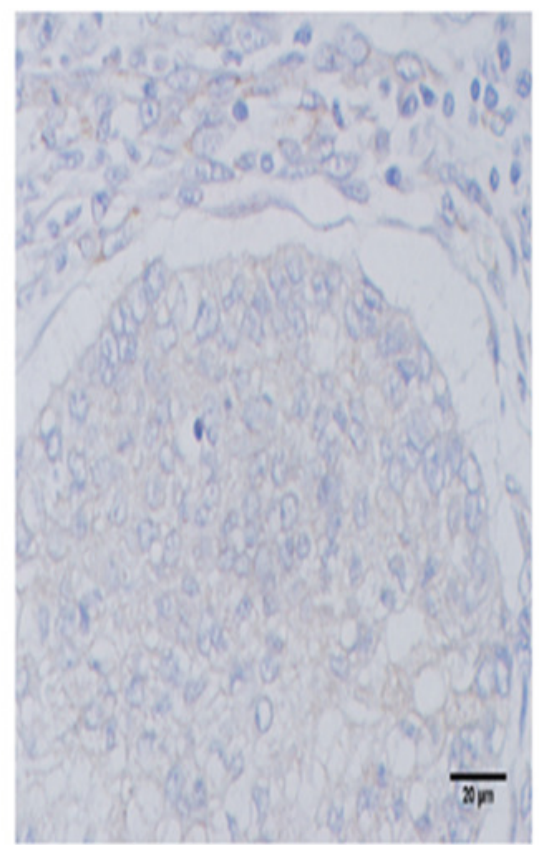

Low expression
C

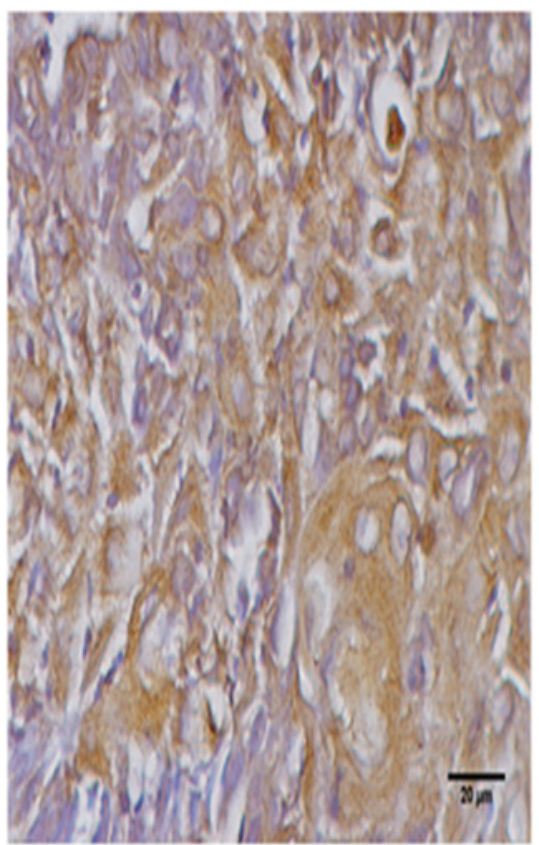

High expression 
Figure 3

Figure 3 The expression of KIAA1199 in LSCC tissues and survival surve.

(A-F) KIAA1199 expression by immunohistochemical staining. (A) Adjacent nonc-ancerous tissue as the negative control. (B) Gastric cancer tissue as the positive control. (C) I stage LSCC tissue. (D) Ilstage LSCC tissue. (E) III stage LSCC tissue. (F) IV stage LSCC tissue. (G) Kaplan-Meier survival curves analysis of ov-erall survival for all patients with KIAA1199 negative and positive LSCC tissue.

A

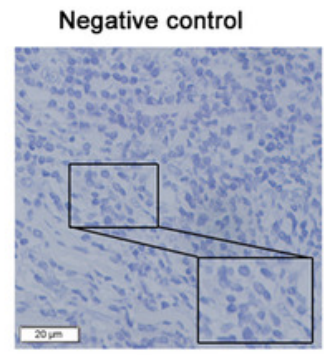

I

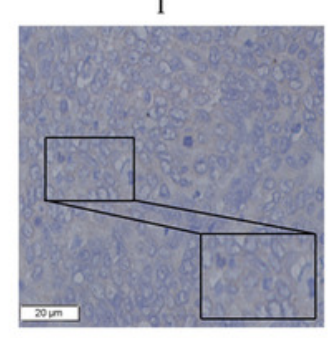

III

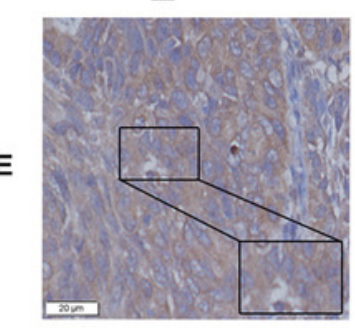

Positive control

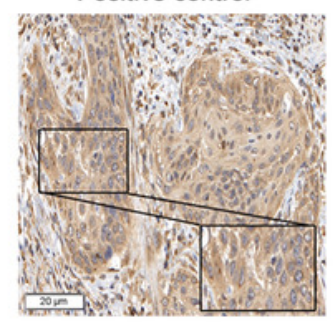

II

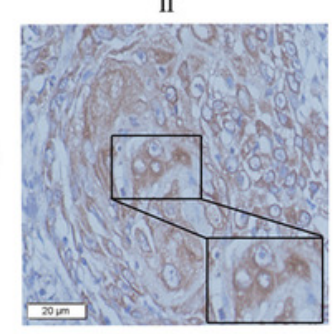

IV

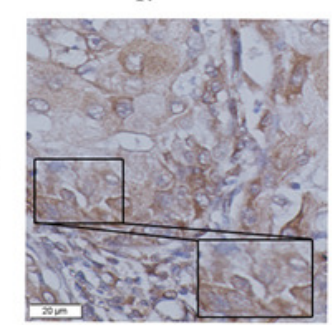

G

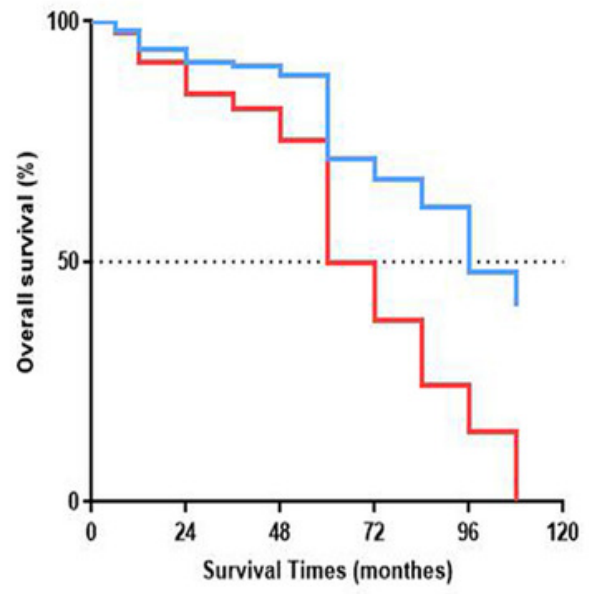

- Low expression

- high expression 


\section{Table $\mathbf{1}$ (on next page)}

Clinicopathological characteristics of patient samples and expression of KIAA1199 in LSCC 
1 Table1: Clinicopathological characteristics of patient samples and expression of KIAA1199 in LSCC

\begin{tabular}{|c|c|c|}
\hline & & Case number / n (\%) \\
\hline \multirow[t]{2}{*}{ Gender } & Male & $103(98.1)$ \\
\hline & Female & $2(1.9)$ \\
\hline \multirow[t]{2}{*}{$\operatorname{Age}(y)$} & $\leq \mathbf{6 0}$ & $44(41.9)$ \\
\hline & $>60$ & $61(58.1)$ \\
\hline \multirow[t]{3}{*}{ Pathologic differentiation } & Poorly & $20(19.05)$ \\
\hline & Moderately & $29(27.62)$ \\
\hline & Highly & $56(53.33)$ \\
\hline \multirow[t]{4}{*}{ Clinic Region } & Supraglottic type & $10(9.52)$ \\
\hline & Trans glottic type & $5(4.76)$ \\
\hline & Glottic type & 87 (82.86) \\
\hline & Subglottic type & $3(2.86)$ \\
\hline \multirow[t]{2}{*}{ T stage } & T1-T2 & $70(66.6)$ \\
\hline & T3-T4 & $35(33.4)$ \\
\hline \multirow[t]{2}{*}{ N stage } & NO & $85(81)$ \\
\hline & N1-N3 & 20 (19) \\
\hline \multirow[t]{3}{*}{ Clinical Stages } & I & $52(49.5)$ \\
\hline & II & $15(14.3)$ \\
\hline & III & $11(10.5)$ \\
\hline
\end{tabular}


IV

Total laryngectomy

The partial laryngetomy

No

Radical cervical clearing

Selective/functional

cleanser

Smoke

Drink

Expression of KIAA1199

High expression neck

Low expression

$27(25.7)$

$28(26.7)$

77 (73.3)

$40(38.1)$

$26(24.8)$

39 (37.1)

Yes

75 (71.4)

$53(50.5)$

52 (49.5)

$50(47.6)$

$55(52.4)$

2 


\section{Table 2 (on next page)}

Correlation between KIAA1199 expression and clinicopathologic characteristics of LSCC patients 
1 Table 2: correlation between KIAA1199 expression and clinicopathologic characteristics of LSCC patients

\begin{tabular}{|c|c|c|c|c|}
\hline \multicolumn{5}{|c|}{ Expression of KIAA1199 (No.) } \\
\hline Gender & Male & $\begin{array}{l}\text { Low } \\
48\end{array}$ & $\begin{array}{l}\text { High } \\
55\end{array}$ & $\begin{array}{c}P \\
0.224\end{array}$ \\
\hline & Female & 2 & 0 & \\
\hline \multirow[t]{2}{*}{ Age(year) } & $\leq 60$ & 21 & 23 & 1.000 \\
\hline & $>60$ & 29 & 32 & \\
\hline \multirow[t]{3}{*}{ Pathologic differentiation } & Poorly & 1 & 19 & $<0.001$ \\
\hline & Moderately & 18 & 11 & \\
\hline & Highly & 31 & 25 & \\
\hline \multirow[t]{4}{*}{ Clinic Region } & Supraglottic type & 3 & 7 & 0.072 \\
\hline & Trans glottic type & 0 & 5 & \\
\hline & Glottic type & 46 & 41 & \\
\hline & Subglottic type & 1 & 2 & \\
\hline \multirow[t]{2}{*}{ T stage } & T1-T2 & 48 & 22 & $<0.001$ \\
\hline & T3-T4 & 2 & 33 & \\
\hline \multirow[t]{2}{*}{$\mathrm{N}$ stage } & No & 50 & 35 & $<0.001$ \\
\hline & N1-N3 & 2 & 18 & \\
\hline \multirow[t]{2}{*}{ Clinical Stage } & I - II & 48 & 19 & $<0.001$ \\
\hline & III-IV & 2 & 36 & \\
\hline \multirow[t]{2}{*}{ Smoke } & No & 18 & 12 & 0.132 \\
\hline & Yes & 32 & 43 & \\
\hline
\end{tabular}




\begin{tabular}{|c|c|c|c|c|}
\hline \multirow[t]{2}{*}{ Drink } & No & 26 & 27 & \multirow[t]{2}{*}{0.846} \\
\hline & Yes & 24 & 28 & \\
\hline \multirow[t]{2}{*}{ Survival status } & survive & 43 & 7 & $<0.001$ \\
\hline & death & 7 & 48 & \\
\hline \multirow[t]{4}{*}{ Survival times (month) } & $\leq 12$ & 2 & 7 & 0.008 \\
\hline & $>12, \leq 36$ & 0 & 8 & \\
\hline & $>36, \leq 60$ & 23 & 22 & \\
\hline & $>60$ & 25 & 18 & \\
\hline
\end{tabular}

2 
Table 3(on next page)

Univariate analyses of various prognostic parameters in patients with LSCC 
1 Table 3: Univariate analyses of various prognostic parameters in patients with LSCC

2

3

\begin{tabular}{|c|c|c|c|}
\hline \multirow{2}{*}{ Parameters } & \multicolumn{3}{|c|}{ Univariate Cox } \\
\hline & Hazard ratio & $95 \% \mathrm{Cl}$ & p-Value \\
\hline Gender & 0.048 & $0-25.791$ & 0.422 \\
\hline $\operatorname{Age}(y)$ & 1.032 & $1.001-1.063$ & 0.040 \\
\hline Pathologic differentiation & 0.643 & $0.524-0.789$ & $<0.001$ \\
\hline Clinic Region & 0.068 & $0.49-1.026$ & 0.068 \\
\hline T stage & 1.402 & $1.139-1.724$ & 0.001 \\
\hline $\mathrm{N}$ stage & 1.679 & $1.148-2.457$ & 0.008 \\
\hline Clinical Stages & 1.445 & $1.180-1.769$ & $<0.001$ \\
\hline Operation & 0.380 & $0.222-0.650$ & $<0.001$ \\
\hline Neck lymph dissection & 0.957 & $0.7106-1.291$ & 0.774 \\
\hline Smoke & 1.028 & $0.560-1.885$ & 0.930 \\
\hline Drink & 0.782 & $0.460-1.330$ & 0.365 \\
\hline expression of KIAA1199 & 12.165 & $5.434-27.233$ & $<0.001$ \\
\hline
\end{tabular}




\section{Table 4(on next page)}

Multivariate analyses of various prognostic parameters in patients with LSCC 
1 Table 4: Multivariate analyses of various prognostic parameters in patients with LSCC

\begin{tabular}{|c|c|c|c|}
\hline \multirow{2}{*}{ Parameters } & \multicolumn{2}{|c|}{ Multivariate Cox } & \multirow[b]{2}{*}{ p-Value } \\
\hline & Hazard ratio & $95 \% \mathrm{Cl}$ & \\
\hline Age(y) & 1.039 & $1.003-1.077$ & 0.035 \\
\hline Clinic Stage & 0.704 & $0.581-0.960$ & 0.023 \\
\hline Operation & 0.285 & $0.093-0.870$ & 0.027 \\
\hline T stage & 0.68 & $0.529-0.874$ & 0.003 \\
\hline Smoke & 0.400 & $0.204-0.785$ & 0.008 \\
\hline expression of KIAA1199 & 27.937 & $10.600-73.632$ & 0.001 \\
\hline
\end{tabular}

2 